\title{
Alteraciones bucales congénitas y del desarrollo en bebés de 0 a 12 meses del Instituto Nacional de Salud del Niño
}

\author{
Congenital and developmental buccal alterations in infants 0 to \\ 12 months of Instituto Nacional de Salud del Niño
}

\begin{abstract}
Resumen
El objetivo del estudio fue determinar la frecuencia de presentación de las alteraciones bucales congénitas y del desarrollo en bebés de 0 a 12 meses de edad atendidos en el Instituto Nacional de Salud del Nińo desde julio a diciembre del 2008. Metodología: previo consentimiento informado de los padres, se realizó el examen clínico estomatológico a 109 bebés, 56 de género masculino y 53 de género femenino. Se utilizó instrumental de examen clínico, una fuente de luz artificial de la unidad dental y una ficha de recolección de datos. Resultados y conclusiones: del total de bebés examinados, el 72,5\% presentaron alteraciones bucales congénitas y del desarrollo. Las prevalentes fueron los nódulos de Böhn con el $40,3 \%$, el frenillo labial medio superior persistente con el 30,2\% y las perlas de Epstein con el 29,3\% de los casos. No se encontraron diferencias significativas respecto al género y grupo etario. Del grupo que presentó alteraciones bucales congénitas y del desarrollo, el 45,9\% nació a término y en el 29,4\% no hubo necesidad de tratamiento. Las estructuras anatómicas más comprometidas fueron los procesos alveolares con el $65,1 \%$ de los casos. Se concluye que las alteraciones bucales congénitas y del desarrollo tienen una alta prevalencia en este grupo etario. Por tal motivo es importante que el odontólogo tenga un vasto conocimiento de éstas, a fin de realizar un diagnóstico temprano, un tratamiento oportuno y un control periódico de su evolución.
\end{abstract}

Palabras clave: Alteraciones bucales congénitas; Alteraciones del desarrollo; Bebes.

\begin{abstract}
The objective of the study was to determine the frecuency of congenital and developmental oral alterations in infants aged 0 to 12 months, of Instituto Nacional de Salud del Niño since July to December 2008. Methodology: prior informed consent of the parents, stomatological examination in 109 infants, 56 male and 53 female, was performed. Instrumental was used in clinical examinations, artificial light source of dental unit and a data collection sheet. Results and conclusions: $72,5 \%$ of the totality examined infant, showed congenital and developmental oral alterations. The prevalent ones were the Böhn's nodules with $40,3 \%$, the persistent upper labial frenulum with 30,2\% and the Epstein's pearls with $29,3 \%$ of cases. No significant differences with respect to gender and age group were found. From the group that presented oral congenital and developmental abnormalities, $45.9 \%$ were born at term and in $29.4 \%$ no need for treatment. The more committed anatomical structures were the alveolar processes $65.1 \%$ of cases. We concluded that oral congenital and developmental abnormalities are highly prevalent in this age group. Therefore it is important for dentists to have a vast knowledge of these, in order to make an early diagnosis, early treatment and regular monitoring of its evolution.

Keywords: Congenital buccal alterations; Developmental alterations; Infants.
\end{abstract}

\section{Introducción}

La integridad del sistema estomatognático en el bebé es importante para cumplir de manera efectiva las funciones de succión, deglución y respiración. Por tal motivo el odontólogo tiene la responsabilidad de diagnosticar las patologías que puedan interferir con éstas funciones.

Actualmente la literatura científica, sobre las alteraciones bucales congénitas y del desarrollo, tiene como referencia a grupos poblacionales diferentes al nuestro. En vista de ello es necesario realizar estudios en la población peruana con el fin de dar a conocer la prevalencia de cada una de éstas.

\section{Artículo Original}

Jean Marcos-Pari', María E. NúñezLizárraga ${ }^{2}$

1. Escuela Académico Profesional de Odontología. Facultad de Odontología de la Universidad Nacional Mayor de San Marcos, Perú.

2. Departamento Académico de Estomatología Pediátrica. Facultad de Odontología de la Universidad Nacional Mayor de San Marcos, Perú.

\section{Correspondencia:}

CD. Jean Marcos Pari.

Calle Los Nardos 113, Lima 28, Perú.

Correo electrónico: jmarcosodonto@gmail.com

Coautora:

Nuñez-Lizárraga: mariael24@yahoo.com

Fecha de recepción: 03-02-14

Fecha de aceptación: 31-10-14

nitas y del desarrollo en 621 bebés de 0 a 6 meses de edad atendidos en el Banco de Leche del Hospital Universitario Materno Infantil en Sáo Luis; donde el $7.25 \%$ de casos presentaron alguna alteración oral, con localización principalmente maxilar. Los quistes de inclusión fueron las alteraciones prevalentes con el $6.28 \%$, presentándose en mayor número en el grupo etario de 0 a 3 meses de edad $^{1}$.

Dinis y col. ${ }^{3}$ en el 2002 (Brasil) evaluaron las características morfológicas y las alteraciones del desarrollo de la cavidad bucal en recién nacidos donde 
el $61.4 \%$ de bebés presentaron procesos alveolares irregulares, el $92.2 \%$ frenillo labial medio superior y el $64 \%$ quistes de inclusión. Concluyendo que los recién nacidos presentan características propias consideradas normales en esa fase etaria ${ }^{2}$.

Palomino y col. ${ }^{3}$ en el 2001 (Perú) determinaron la distribución de perlas de Epstein en 235 recién nacidos en el Instituto Nacional Materno Perinatal donde se encontró una alta prevalencia del $73,61 \%$; siendo ligeramente mayor en el género femenino ${ }^{3}$.

Baldani y col. ${ }^{4}$ en 1999 (Brasil) estudiaron la prevalencia de alteraciones bucales en 200 bebés de 0 a 24 meses atendidos en las clínicas públicas de bebés de Ponta Grossa donde encontraron que el $21 \%$ de bebés presentaron alteraciones bucales, el grupo etario de 0 a 3 meses presentó una mayor prevalencia con un $26,98 \%$, las alteraciones prevalentes fueron los quistes de inclusión con un $35,71 \%$. Se concluyó además que no hubo necesidad de tratamiento para el $76,19 \%$ de bebes que presentaron estas alteraciones bucales ${ }^{4}$.

Côrrea y col. ${ }^{6}$ en 1997 (Brasil) identificaron las alteraciones bucales del desarrollo y de la erupción dentaria en 70 recién nacidos donde el $41.1 \%$ presentaron quistes de inclusión, siendo el $28.5 \%$ nódulos de Böhn y el $12.9 \%$ perlas de Epstein. El 1.4\% de bebés presentaron un diente natal 5 .

Acevedo y col. en 1995 (México) investigaron la prevalencia de quistes de inclusión en 50 recién nacidos del hospital Materno Infantil Iguarán del Distrito Federal, siendo del 44\%. La entidad que se observó con mayor predominio fue el nódulo de Böhn. ${ }^{6}$

Ponce y col. ${ }^{7}$ en 1994 (México) examinaron 1591 neonatos en el Hospital General Iztapalapa de la Comunidad

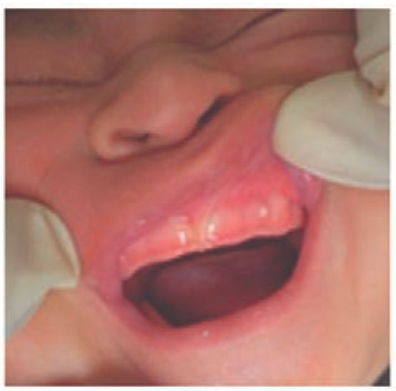

Fig.1.

Rodete fibroso de Robin y Magitot
Económica Europea, donde el 40\% de recién nacidos presentaron quistes de inclusión, el 33.5\% quistes alveolares, el $17 \%$ quistes palatinos, el $1 \%$ quistes de erupción y el $0.1 \%$ quistes de la lamina dental, sin predominio en el género. Se encontró además 5 dientes neonatales en tres neonatos, representando el $0.1 \%$ de casos ${ }^{7}$.

Díaz y col.8 en 1993 (México) realizaron un estudio en 1200 neonatos nacidos en el Instituto Nacional de Perinatología, donde el $46 \%$ presentaron quistes de inclusión, el $2.5 \%$ quistes de erupción y el $0.1 \%$ dientes natales ${ }^{8}$.

Watson ${ }^{9}$ en 1985 (Perú) determinó la prevalencia de condiciones orales en 1000 neonatos nacidos en el Hospital General Base Cayetano Heredia y en la Maternidad de Lima, siendo del $98.2 \%$. Donde las más predominantes fueron las perlas de Epstein con el $92 \%$, los linfangiomas con el $74 \%$, los leucoedemas con el $24 \%$ y las úlceras pterigoideas con el $2 \%$. Se identificó también una prevalencia del $0.7 \%$ para los dientes neonatales y del $0.1 \%$ para los dientes natales?

El objetivo de la presente investigación fue determinar las alteraciones bucales congénitas y del desarrollo en bebés de 0 a 12 meses de edad atendidos en el Instituto del Niño desde julio a diciembre del 2008, según el género, el grupo etario, la edad gestacional al nacer, la necesidad de tratamiento y su distribución anatómica. Este estudio servirá de base para posteriores investigaciones enfocadas en la prevención y tratamiento de las alteraciones bucales congénitas y del desarrollo de este grupo etario.

\section{Materiales y método}

El estudio fue descriptivo y de corte transversal. La población estuvo constituida por pacientes bebés de 0 a 12 meses de edad, provenientes de Lima, atendidos en el Instituto Nacional de Salud del Niño. El tipo de muestreo fue no probabilístico, por conveniencia. La muestra estuvo conformada por 109 bebés ( 56 de genero masculino y 53 de genero femennino) de 0 a 12 meses de edad atendidos en el servicio de Odontología del Bebé en el Instituto Nacional de Salud del Niño desde julio a diciembre del ańo 2008. Los criterios de inclusión fueron bebés sin enfermedad sistémica y que no recibieron alguna intervención que altere la cavidad bucal.

Previo consentimiento informado de los padres, se solicitó ayuda a la madre para que se ubique en el sillón dental y colocara al bebé sobre su vientre en posición decúbito dorsal. Seguidamente se procedió a realizar la higiene oral del bebé con ayuda de una gasa y agua estéril. Después se realizó el examen clínico estomatológico utilizando guantes para examen, espejos bucales y una fuente de luz artificial de la lámpara de la unidad dental. El protocolo durante el examen clínico bucal fue: evaluación de los labios, los carrillos, el paladar duro, el paladar blando, la orofaringe, la lengua, el piso de boca, los procesos alveolares, las encías y los dientes. Se utilizó una ficha de recolección de datos en la cual se registró la información general del paciente y las alteraciones bucales congénitas y del desarrollo que presentaban.

La recolección de datos se realizó con la autorización de la Dirección Ejecutiva de Apoyo a la Investigación y Docencia Especializada del Instituto Nacional de Salud del Niño. Para el procesamiento de los datos obtenidos se utilizó el programa SPSS versión 12.0 con el cual se realizó un análisis multivariado para describir la distribución de la muestra con los cruces respectivos de cada variable de acuerdo a los objetivos planteados.

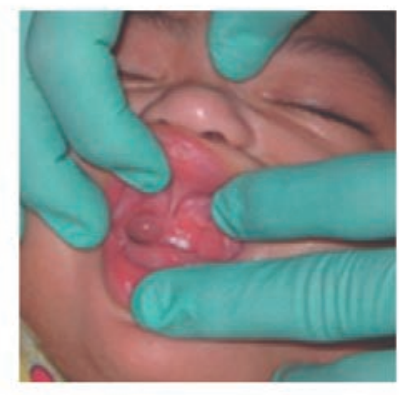

Fig.2.

Quiste de erupción

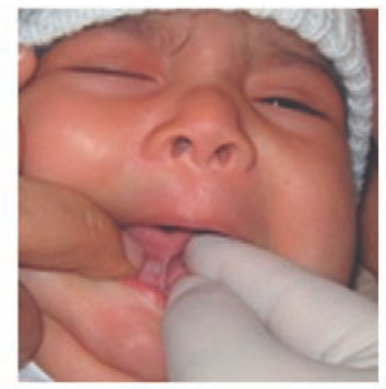

Fig.3.

Anquiloglosia por frenillo lingual. 


\section{Resultados}

Tabla 1. Distribución de la muestra según tipo de alteraciones bucales congénitas y del desarrollo

\begin{tabular}{lcc}
\hline TIPO DE ALTERACION & $\mathbf{n}$ & $\%$ \\
\hline Nódulos de Böhn & 44 & 40.3 \\
Frenillo labial medio superior persistente & 33 & 30.2 \\
Perlas de Epstein & 32 & 29.3 \\
Hipoplasia de esmalte & 12 & 11.0 \\
Anquiloglosia & 08 & 7.3 \\
Quiste de la lámina dental & 07 & 6.4 \\
Dientes natales & 03 & 2.7 \\
Dientes neonatales & 02 & 1.8 \\
Ulcera de Riga-fede & 02 & 1.8 \\
Hematoma de erupción & 01 & 0.9 \\
Quiste de erupción & 01 & 0.9 \\
Micrognasia mandibular & 01 & 0.9 \\
Epulis congénito del recién nacido & 00 & 0.0 \\
\hline
\end{tabular}

Tabla 2. Distribución de la muestra según la presencia de alteraciones bucales congénitas y del desarrollo y género

\begin{tabular}{lccccccc}
\hline Presencia de alteraciones & \multicolumn{2}{c}{ Masculino } & \multicolumn{2}{c}{ Femenino } & \multicolumn{2}{c}{ Total } \\
bucales congénitas y del desarrollo & $\mathbf{n}$ & $\%$ & & $\mathbf{n}$ & $\%$ & $\mathrm{n}$ & $\%$ \\
\hline No presentan & 15 & 13.8 & 15 & 13.8 & 30 & 27.5 \\
Presentan & 41 & 37.6 & 38 & 34.9 & 79 & 72.5 \\
Total & 56 & 51.4 & 53 & 48.6 & 109 & 100 \\
\hline
\end{tabular}

Tabla 3. Distribución de la muestra según la presencia de alteraciones bucales congénitas y del desarrollo y grupo etario

\begin{tabular}{lcccccccccc}
\hline Presencia de alteraciones & \multicolumn{1}{c}{$\mathbf{0}-1$ mes } & \multicolumn{2}{c}{$1-3$ meses } & \multicolumn{2}{c}{$4-6$ meses } & \multicolumn{2}{c}{ 7-12 meses } & \multicolumn{3}{c}{ Total } \\
Bucales congénitas y del desarrollo & $\mathrm{n}$ & $\%$ & $\mathrm{n}$ & $\%$ & $\mathrm{n}$ & $\%$ & $\mathrm{n}$ & $\%$ & $\mathrm{n}$ & $\%$ \\
\hline No presentan & 09 & 8.3 & 04 & 3.7 & 07 & 6.4 & 10 & 9.2 & 30 & 27.5 \\
Presentan & 22 & 20.2 & 20 & 18.3 & 16 & 14.7 & 21 & 19.3 & 79 & 72.5 \\
Total & 31 & 28.4 & 24 & 22.0 & 23 & 21.1 & 31 & 28.4 & 109 & 100 \\
\hline
\end{tabular}

Tabla 4. Distribución de la muestra según la presencia de alteraciones bucales congénitas y del desarrollo y edad gestacional al nacer

\begin{tabular}{|c|c|c|c|c|c|c|c|c|}
\hline \multirow[t]{2}{*}{$\begin{array}{l}\text { Presencia de alteraciones Buca- } \\
\text { les congénitas y del desarrollo }\end{array}$} & \multicolumn{2}{|c|}{ Pre término } & \multicolumn{2}{|c|}{ A término } & \multicolumn{2}{|c|}{$\begin{array}{l}\text { Post } \\
\text { término }\end{array}$} & \multicolumn{2}{|c|}{ Total } \\
\hline & $\mathrm{n}$ & $\%$ & $\mathrm{n}$ & $\%$ & $\mathrm{n}$ & & $\mathrm{n}$ & $\%$ \\
\hline No presentan & 02 & 01.8 & 25 & 22.9 & 03 & 2.8 & 30 & 27.5 \\
\hline Presentan & 22 & 20.2 & 50 & 45.9 & 07 & 6.4 & 79 & 72.5 \\
\hline Total & 24 & 22.0 & 75 & 68.8 & 10 & 9.2 & 109 & 100 \\
\hline
\end{tabular}

Tabla 5. Distribución de la muestra según la presencia de alteraciones bucales congénitas y del desarrolloy necesidad de tratamiento

\begin{tabular}{|c|c|c|c|c|c|c|c|}
\hline \multirow[t]{2}{*}{$\begin{array}{l}\text { Presencia de alteraciones Buca- } \\
\text { les congénitas y del desarrollo }\end{array}$} & \multicolumn{2}{|c|}{$\begin{array}{l}\text { Sin necesidad } \\
\text { de tratamiento }\end{array}$} & \multicolumn{2}{|c|}{$\begin{array}{l}\text { Observación y } \\
\text { control }\end{array}$} & \multicolumn{2}{|c|}{$\begin{array}{l}\text { Con necesidad } \\
\text { de tratamiento }\end{array}$} & Total \\
\hline & $\mathrm{n}$ & $\%$ & $\mathrm{n}$ & $\%$ & $n$ & $\%$ & n $\%$ \\
\hline No presentan & 30 & 27.5 & 00 & 0.0 & 00 & 0.0 & $30 \quad 27.5$ \\
\hline Presentan & 32 & 29.4 & 23 & 21.1 & 24 & 22.0 & 7972.5 \\
\hline Total & 62 & 56.9 & 23 & 21.1 & 24 & 22.0 & 109100 \\
\hline
\end{tabular}


Tabla 6. Distribución anatómica de las alteraciones bucales congénitas y del desarrollo

\begin{tabular}{lrr}
\hline $\begin{array}{l}\text { Presencia de alteraciones bucales } \\
\text { Congénitas y del desarrollo }\end{array}$ & $\mathrm{n}$ & $\%$ \\
\hline Procesos alveolares & 40 & 42.2 \\
Labios & 33 & 30.3 \\
Paladar duro & 28 & 25.7 \\
Paladar blando & 19 & 17.4 \\
Dientes & 14 & 12.8 \\
Piso de boca & 08 & 7.3 \\
Lengua & 02 & 1.8 \\
Carrillos & 00 & 0.0 \\
Orofaringe & 00 & 0.0 \\
Encía & 00 & 0.0 \\
\hline
\end{tabular}

Del total de 109 bebés examinados, 79 bebés presentaron alteraciones bucales congénitas y del desarrollo $(72,5 \%)$, de las cuales las más frecuentes fueron los nódulos de Böhn (40,3\%), el frenillo labial medio superior persistente $(30.2 \%)$, y las perlas de Epstein (29,3\%). (Tabla 1)

Se determinó que, del grupo que presentó alteraciones bucales congénitas y del desarrollo, no existieron diferencias entre el género masculino $(37,6 \%)$ y el femenino $(34,9 \%)$. (Tabla 2)

Se evidenció que el grupo etario menor a 1 mes presentó una mayor frecuencia de alteraciones bucales congénitas y del desarrollo $(20,2 \%)$; seguido por el grupo etario de 7 a 12 meses $(19,3 \%)$ y el grupo etario de 1 a 3 meses (18,3\%). (Tabla 3)

Se encontró que el grupo de bebés nacidos a término presentó una mayor frecuencia de alteraciones bucales congénitas y del desarrollo (45,9\%), seguido por el grupo nacido pretérmino $(20,2 \%)$ y el grupo nacido postérmino $(6,4 \%)$. (Tabla 4 )

Se observó que en la mayoría de alteraciones bucales congénitas y del desarrollo no hubo necesidad de tratamiento $(29,4 \%)$, seguido por el grupo con necesidad de tratamiento (22\%) y el grupo de observación y control $(21,1 \%)$. (Tabla 5)

Se determinó que las estructuras anatómicas mas afectadas por las alteraciones bucales congénitas y del desarrollo fueron los procesos alveolares $(42,2 \%)$, seguido por los labios $(30,3 \%)$ y el paladar duro $(25,7 \%)$. (Tabla 6)

\section{Discusión}

En el presente estudio se reportó una marcada frecuencia de alteraciones bucales congénitas y del desarrollo $(72,5 \%)$. Este hallazgo es menor a los resultados obtenidos en Perú por Watson $^{9}(98.2 \%)$ y mayor a los obtenidos por Baldani ${ }^{4}(21 \%)$ y Santos ${ }^{1}$ (7.25\%) en Brasil. Es importante resaltar que la mayoría de bebés presentó más de una alteracion bucal congénita y del desarrollo (44\%), a diferencia de los resultados obtenidos por Watson ${ }^{9}$ (92\%) que fueron mayores.

En esta investigación no se evidenciaron diferencias con respecto al género, coincidiendo con Watson'. Sin embargo, en Brasil Baldani ${ }^{4}$ encuentra un ligero predominio en el género femenino. Con respecto a los quistes de inclusión, se identificó un ligero predominio en el género femenino; coincidiendo con los hallazgos de Baldani ${ }^{4}$ y Palomino ${ }^{3}$ en Perú.

En relación al grupo etario, se identificó que los quistes de inclusión se presentaron en mayor proporción en el grupo etario menor de 1 mes, disminuyendo considerablemente conforme el grupo etario era mayor, coincidiendo con los trabajos realizados por Santos ${ }^{1}$, Baldani ${ }^{4}$, Walter ${ }^{10}$ en Brasil y Martin ${ }^{11}$ en Argentina. Sin embargo, se registraron quistes de inclusión en el grupo de 7 a 12 meses, contradiciendo a estos cuatro autores debido a que sostienen que estos quistes desaparecen entre los 3 a 4 primeros meses de edad.

En lo concerniente a la edad gestacional al nacer, los hallazgos de este estudio son importantes debido a que no se han reportado estudios similares que relacionen esta variable con las alteraciones bucales congénitas y del desarrollo.

Con respecto a la necesidad de tratamiento, estos hallazgos son importantes debido a que sólo tenemos como referencia el estudio realizado por Baldani ${ }^{4}$ el cual es mayor en el grupo de observación y control.

En relación a la distribución anatómica de las alteraciones bucales congénitas y del desarrollo, se coincidió con el estudio de Watson que indica que las estructuras anatómicas más afectadas son el paladar duro y los procesos alveolares, es decir aquellas zonas mucosas soportadas por tejido óseo.

\section{Conclusiones}

- Las alteraciones bucales congénitas y del desarrollo más frecuentes fueron los nódulos de Böhn con el
$40.3 \%$, el frenillo labial medio superior persistente con el $30.2 \%$ y las perlas de Epstein con el 29,3\%.

- No existieron grandes diferencias con respecto al género y grupo etario.

- Se determinó que el $45 \%$ de bebés que presentaron alguna alteracion bucal congénita y del desarrollo nació a término.

- Se reportó que el 29,4\% de bebés que presentaron alguna alteraciones bucales congénitas y del desarrollo no hubo necesidad de tratamiento.

- Las estructuras anatómicas que presentaron una mayor frecuencia de alteraciones bucales congénitas y del desarrollo fueron los procesos alveolares con el $42,2 \%$, seguida por los labios con el 30,3\% y el paladar duro con el 25,7\%.

\section{Referencias bibliográficas}

1. Santos FF, Pinho JR, Libério SA, Cruz MC. Prevalência de alteraçóes orais congênitas e de desenvolvimento em bebês de 0 a 6 meses. Rev Odonto Ciênc. 2009;24(1):77-80.

2. Dinis MG, Ciamponi AL, Correa MS. Características morfológicas da cavidade bucal dos recém-nascidos. RPG rev. posgrad. 2002;9(2):155-60.

3. Palomino M, Aguilar D. Perlas de Epstein, su distribución y frecuencia en recién nacidos vivos. Informe Científico. Ceo.com. pe [Internet]. [Consultado 2008 mar 13]. Disponible en: http:// www.ceo.com.pe/005_revista_ art04.htm

4. Baldani MH, Lopes CM, Scheidt WA. Prevalência de alteraçóes bucais em crianças atendidas nas clínicas de bebês públicas de Ponta Grossa - PR, Brasil. Pesqui Odontol Bras. 2001; 15(4):302$307 .<$ doi: $10.1590 /$ S 1517 $74912001000400006>$.

5. Côrrea MS, Villena RS, Frascino SM. Características da cavidade bucal e ocorrência de anomalias em recém-nascidos. Rev Paul Odontol. 1997;19(3):3440.

6. Acevedo GO, Castillo CDS, De La Teja AE. Prevalencia de quistes de inclusión en recién nacidos. Rev ADM 1995;52(6):291-292. 
7. Ponce DM, Bustillos CM, Gómez TL, Fernández LC, Rubí ML. Alteraciones congénitas bucales neonatales. Bol Méd Hosp Infant Méx. 1994;51(8):540-2.

8. Díaz RR, Gutiérrez OJ, Shor HF, Benítez TC. Características bucomaxilofaciales del recién nacido sano. Perinatol Reprod Hum. 1993;7(3):114-9.

9. Watson BM. Estudio clínico de la prevalencia de condiciones orales en 1000 neonatos nacidos en el Hospital Base Cayetano Heredia. [Tesis bachiller] Lima: Universidad Peruana Cayetano Heredia; 1985.

10. Figueiredo WL; Ferelle A; Issao M. Odontología para el bebé. Odontopediatría desde el nacimiento hasta los 3 años. $1^{\text {a }}$ ed. Sao Paulo: Actualidades Médico Odontológicas Latinoamérica. 2000. 45-70p.

11. Martín KN. Lesiones estomatológicas desde el nacimiento hasta los tres años. Bol AAON. 2002;31(1):3-11.

12. Martinez VG. Dientes natales y neonatales. Acta Odontológica Venezolana [Internet]. 1997 [Consultado 2008 abr 15]; 35(2). Disponible en: http:// www.actaodontologica.com/ ediciones/1997/2/dientes_ natales_neonatales_1.asp

13. Rioboo CM, Planells PP, Rioboo GR. Epidemiología de la patología de la mucosa oral más frecuente en niños. Med
Oral Patol Oral Cir Bucal. 2005; 10(5):376-87.

14. Cameron A; Widmer RP. Manual de odontología pediátrica. $1^{\text {a }}$ ed. Madrid: Hartcourt Brace; 1998. 143-219p.

15. Côrrea MS, Abanto J, Pires FN, Acevedo G. Anquiloglosia y amamantamiento: revisión y reporte de caso. Rev Estomatol Herediana. $2008 ; 18(2): 123-127$.

16. Mosby. Diccionario Medicina, Enfermería y Ciencias de la Salud. $5^{a}$ ed. Madrid: Editorial Harcot; 2000.

17. Gomela TL, Cunningham D, Eyal FG, Zenk KE. Neonatología. $5^{a}$ ed. Buenos Aires: Editorial médica Panamericana; 2006. 23-37p. 